\section{Spinal tap}

FollowING the linkage of the common neurodegenerative disease spinal muscular atrophy (SMA) to the long arm of chromosome 5 last year, efforts are underway to identify the abnormal gene(s). The most intriguing candidate to emerge thus far is described by $L$. $L$. Lien et al. (Proc. natn. Acad. Sci. U.S.A. 88, 7873-7876; 1991), who used antibodies to dystrophin to isolate related genes including that of microtubule-associated protein-1B, which encodes a large cytoskeletal protein highly expressed in the anteriorhorn motor neurons affected in SMA. Tight linkage was found between the SMA locus and MAP-1B, but two small chronic SMA families possessed recombinants. At worst, then, MAP-1B is a very close marker for SMA; at best, given the possibility of nonallelic heterogeneity in the chronic disease, it may have a more direct involvement in some cases.

\section{Suspended animation}

WHEN matter and antimatter meet, the normal expectation is that they immediately annihilate one another. So it came as something of a surprise to $\mathrm{T}$. Yamazaki et al. two years ago to find that negative kaons can survive as long as $10 \mathrm{~ns}$ after arriving and stopping in liquid helium, before being pulled electrostatically into the host atoms' positive nuclei. Yamazaki and colleagues have now repeated the experiment with antiprotons, which (unlike kaons) have an infinite natural lifetime when isolated from ordinary matter (M. Iwasaki et al. Phys. Rev. Lett. $67,1246-1249 ; 1991$ ). Although 96 per cent of the incident antiprotons in their sample spiralled immediately into helium nuclei, the remainder enjoyed a kind of suspended animation of up to 15 $\mu s$, held, apparently in some metastable quasiatomic state, before meeting their inevitable doom.

\section{On the height side}

A WIDENING gulf in the healthiness of rich and poor in industrialized nations may be read into adult death rates, points out J. P. Mackenbach (The Lancet 338, $764 ; 1991)$, but other indicators reveal a brighter picture. Comparison of two surveys carried out in Holland in 1964 66 and 1980 indicates that the disparity in height between children of different socioeconomic groups again narrowed, continuing a trend seen since the turn of the century; for example, whereas in boys aged 11-16 the difference was $29 \mathrm{~mm}$ a quarter of a century ago, in 1980 it was $14 \mathrm{~mm}$. Use of height may be an indirect indicator says Mackenbach. But he hints that those who consider the welfare state to have been unsuccessful in health care, given the adult mortality data, might care to think again.
That the mutagenic approach used here resembles that employed a decade earlier in Drosophila, to powerful effect, is not surprising: Jürgens, the senior author of these papers, was a postdoctoral fellow in the group that performed the saturation of the Drosophila genome for embryo pattern mutations ${ }^{5,6}$. The ready application of Drosophila genetics to Arabidopsis again confirms Whyte's foresight, 45 years ago, in calling Arabidopsis a "botanical Drosophila" . Indeed, mutagenesis of this sort is even simpler in Arabidopsis than in Drosophila, because the plant can selffertilize, allowing homozygosis of newly induced mutations in a single generation. This is only practical for sex-linked mutations in Drosophila.

The original detailed observations of embryonic cleavage patterns were made on plants $^{8}$, using Capsella, a relative of Arabidopsis. These were later followed by similar observations in animals ${ }^{9}$. Indeed, some of the first interpretations of cleavage patterns in animal embryogenesis derived from earlier con-

\section{COSMOLOGY}

\section{Texture and cosmic structure}

\section{Joseph Silk and Roman Juszkiewicz}

AMONG the fundamental questions of cosmology are how the original hot primordial soup created in the Big Bang collapsed to become the galaxies we see today and why these are distributed with the particular degree of order that they have. One solution, proposed ${ }^{1}$ by Neil Turok in 1989 following an earlier suggestion by Davis ${ }^{2}$, was the notion of 'texture', a kind of froth that pervades the Universe and which would have arisen during the first moments following the Big Bang. In a series of papers ${ }^{3-\phi}$, Turok and colleagues now show that texture could produce the observed spectrum of galactic masses and their spatial distribution. Nevertheless, provocative questions remain to be answered concerning this explanation for large-scale structure.

Global texture is a relic of the phase transition that demarcated the end of the grand-unification era, during which the nuclear and electromagnetic forces behaved as different aspects of a single force. An energy field, the Higgs field, was responsible for the grand-unification process when newly created, very massive particles were present that carried the fundamental forces of nature, the nuclear, weak and strong, and the electromagnetic forces. As the Universe cooled below the grand-unification energy scale (about $10^{16} \mathrm{GeV}$ ), the uniform part of the Higgs field, occupying most of space, decayed, leaving behind fun- clusions on plant cell-division patterns ${ }^{10}$. With the application of saturation genetics and molecular cloning methods to reveal the mechanisms of pattern formation in plant embryos, plants may regain their position as the primary source of knowledge about patterned cell divisions in multicellular organisms.

Elliot M. Meyerowitz is in the Division of Biology, California Institute of Technology, Pasadena, California 91125, USA.

1. Mayer, U., Torres Ruiz, R. A., Berleth, T., Miséra, S. \& Jürgens, G. Nature 353, 402-407 (1991)

2. Jürgens, G., Mayer, U., Torres Ruiz, R. A., Berleth, T. \& Miséra, S. Development 91 (Suppl. 1) 27-38 (1991)

3. Schneider, T., Dinkins, R., Robinson, K., Shellhammer J. \& Meinke, D. W. Devl Biol. 131, 161-167 (1989).

4. Patton, D. A., Franzmann, L. H. \& Meinke, D. W. Molec gen. Genet. 227, 337-347 (1991)

5. Jürgens, G., Weischaus, E., Nüsslein-Volhard, C. \& Kluding, H. Wilhelm Roux Arch. dev. Biol. 193, 283 295 (1984)

6. Wieschaus, E., Nüsslein-Volhard, C. \& Jürgens, G Wilhelm Roux Arch. dev. Biol. 193, 296-307 (1984).

7. Whyte, R. O. Crop Production and Environment (Faber \& Faber, London, 1946)

8. Hanstein, J. Bot. Abhandf., Bonn 1, 1-112 (1870).

9. Whitman, C. Q. JI microsc. Sci. 18, 215-315 (1878)

10. Wilson, E. B. The Cell in Development and Inheritance 265 ff. (Macmillan, New York, 1896)

11. Schaffner, M. Ohio Nat. 7, 1-8 (1906) damental particles such as quarks and gluons that carry the strong force. However, occasional non-uniformities in the Higgs field survived as topological defects of 'knots' that are infinitesimal $\left(\sim 10^{-26} \mathrm{~cm}\right)$ regions of relic energy density that cannot decay.

\section{Defects}

Texture is not the only topological defect that can be created by phase transitions in the early Universe. Zero-dimensional, point-like monopoles, one-dimensional strings and two-dimensional walls have all been studied, but none has been shown compellingly to lead to a successful theory of large-scale cosmological structure. But the non-uniform Higgs field represented by texture results in three-dimensional knots with an energy density that generates density perturbations with just the right largescale distribution to be of interest to cosmologists. Once the horizon scale (the distance travelled by light since creation) becomes comparable to the radius of a texture, the texture collapses owing to the tension associated with the deformation of the Higgs field. As it collapses, it drags in with it all of the associated energy density.

When it reaches a size of about $10^{-30}$ $\mathrm{cm}$, the knot unwinds itself, emitting a burst of weakly interacting massless particles known as Goldstone bosons. The energy density associated with the 